\title{
PESSOAS LGBTI+ E A COVID-19: PARA PENSARMOS QUESTÕES SOBRE SAÚDE
}

\author{
THE LGBTI+ PEOPLE AND COVID-19: \\ FOR US TO THINK ABOUT HEALTH ISSUES
}

\section{PERSONAS LGB'TI+ Y LA COVID-19: PARA PENSAR EN CUESTIONES DE SALUD}

\author{
Anderson Reis Sousa ${ }^{1}$ \\ Caio Felipe Campos Cerqueira ${ }^{2}$ \\ Carle Porcino ${ }^{3}$ \\ Kleber José Fonseca Simões ${ }^{4}$
}

\begin{abstract}
Como citar esse artigo: Sousa AR, Cerqueira CFC, Porcino C, Simões KJF. Pessoas lgbti+ e a covid-19: para pensarmos questões sobre saúde. Rev baiana enferm. 2021;35:e36952.

Objetivo: refletir teórica e criticamente o cenário da situação de saúde de pessoas LGBTI+ frente ao Covid-19 em contexto de pandemia no Brasil. Método: Estudo teórico e reflexivo estruturado a partir do arcabouço teórico e analítico de gênero e dos achados empíricos sobre a pandemia do novo Coronavírus, causadora da Covid-19. Realizou-se a decomposição não estruturada dos achados publicados na mídia digital e nas bases de dados científicos sobra a Covid-19, bem como a interseção com a saúde de pessoas LGBTI+, especialmente no Brasil. Resultados: Há repercussões negativas geradas pela Covid-19 à saúde de pessoas LGBTI+ que são intensificadas por ações biopolíticas determinantes de exposição humana à vulnerabilidade, negação de direitos, discriminação, violências e iniquidades, que potencializam a degradação da saúde e a condição humana. Conclusão: O surgimento da Covid-19 precipita e intensifica as vulnerabilidades e iniquidades em saúde de pessoas LGBTI+, conduzindo-as à marginalização e ao risco expressivo à vida.
\end{abstract}

Descritores: Pandemias. Infecções por Coronavírus. Minorias Sexuais e de Gênero. Gênero. Diversidade de Gênero.

Objective: to reflect theoretically and critically the scenario of the bealth situation of LGBTI+ people in face of the Covid-19 in the context of a pandemic in Brazil. Method: A theoretical and reflective study structured on the theoretical and analytical framework of gender and empirical findings on the pandemic of the new Coronavirus, causing the Covid-19. It was carried out the unstructured decomposition of findings published in digital media and scientific databases on Covid-19, as well as the intersection with the health of LGBTI+ people, especially in Brazil. Results: There are negative repercussions generated by Covid-19 on the health of LGBTI+ people, which are intensified by biopolitical actions that determine human exposure to vulnerability, denial of rights, discrimination, violence and inequalities, which potentialize health degradation and the human condition. Conclusion: The appearance of Covid-19 precipitates and intensifies the vulnerabilities and inequities in the health of LGBTI+ people, leading them to marginalization and expressive risk to life.

Descriptors: Pandemics. Coronavirus infections. Sexual and Gender Minorities. Gender. Gender Diversity.

\footnotetext{
Enfermeiro. Mestre em Enfermagem. Docente da Universidade Federal da Bahia. Salvador, Bahia, Brasil. son.reis@hotmail.com. https://orcid.org/0000-000I8534-1960.

2 Licenciado em Ciências sociais. Mestre em Ciências Sociais. Docente do Instituto Federal do Rio Grande do Sul. Canoas, Rio Grande do Sul, Brasil. https://orcid. org/0000-0002-1 I68-6525.

3 Psicóloga. Mestre em Estudos Interdisciplinares. Universidade Federal da Bahia. https://orcid.org/0000-000 I-6392-029 |

${ }^{4}$ Historiador. Mestre em História Social. Docente da Universidade do Estado da Bahia. Conceição do Coité, Bahia, Brasil. https://orcid.org/0000-0002-884I-8553.
} 
Objetivo: reflejar teórica y críticamente el escenario de la situación de salud de las personas LGBTI+ frente al Covid-19 en el contexto de una pandemia en el Brasil. Método: Un estudio teórico y reflexivo estructurado en el marco teórico y analítico del género y los hallazgos empíricos sobre la pandemia del nuevo Coronavirus, que causa el Covid-19. Se realizó la descomposición no estructurada de los hallazgos publicados en los medios digitales y en las bases de datos científicas sobrantes de Covid-19, asi como la intersección con la salud de las personas LGBTI+, especialmente en Brasil. Resultados: Hay repercusiones negativas generadas por el Covid-19 en la salud de las personas LGBTI+, que se intensifican por las acciones biopoliticas que determinan la exposición humana a la vulnerabilidad, la negación de derechos, la discriminación, la violencia y las desigualdades, que potencian la degradación de la salud y la condición humana. Conclusión: La aparición del Covid-19 precipita e intensifica las vulnerabilidades y desigualdades en la salud de las personas LGBTI+, lo que las lleva a la marginación y a un riesgo significativo para la vida.

Descriptores: Pandemias. Infecciones por el Coronavirus. Minorías sexuales y de género. Género. Diversidad de género.

\section{Introdução}

A atual pandemia ocasionada pelo vírus SARS-CoV-2, geradora da doença conhecida como Covid-19, tem afetado o estado de saúde de milhares de pessoas em todo o planeta ${ }^{(1)}$. Seja a partir da infecção direta ocasionada pelo vírus, que em seus casos extremos acarreta o acometimento do corpo em suas funções respiratórias e demais componentes orgânicos, e/ou através de processos coletivos de adoecimento psíquico que condições de distanciamento e/ou isolamento social podem impor.

Pessoas em situações de vulnerabilidade tendem a agudizar processos de subjetivação e sofrimento devido a situações como a que estamos vivendo $^{(2)}$. Nesse sentido, a desigualdade estrutural que dá régua e compasso às tramas das relações sociais cotidianas, sempre coloca em posição de maior vulnerabilidade as pessoas mais pobres, menos escolarizadas, sem acesso à informação e aquelas que estão imersas em lógicas de subalternidades interseccionais, fundadas nos eixos de opressão que comportam os marcadores sociais da diferença relacionados a cor, raça/etnia, sexo, gênero, identidade de gênero, orientação sexual, idade, deficiência, classe social e outros.

Por conseguinte, dados do relatório pioneiro da OutRight Action International evidenciam os impactos e efeitos da pandemia da Covid-19 em pessoas Lésbicas, Gays, Bissexuais, Transgêneras, Queers e Intersexuais (LGBTQI) de 38 países, ao colocar em evidência que os desafios enfrentados por esse segmento tanto em relação ao vírus quanto a perda econômica se sobrepõem quando comparadas a população em geral $^{(3)}$. Dentre os principais desafios apontados pela OutRight estão: a devastação dos meios de subsistência, resultante do aumento da insegurança alimentar e perda de abrigo; efeito da perda de empregos e queda econômica; interrupções no acesso aos cuidados de saúde, incluindo medicamentos essenciais para o HIV e transição; bem como a relutância em procurar atendimento de saúde devido à discriminação, enfrentada mesmo fora de uma epidemia; colocando em evidência o risco elevado de violência doméstica e familiar em virtude de ser a forma mais prevalente de violência enfrentada por esse segmento; além disso, há ainda o isolamento social e o aumento da ansiedade, que são mais intensificados decorrente a exclusão familiar e social.

Desse modo, pensar sobre o atual contexto pandêmico e as vidas de pessoas LGBTI+ é fundamental para analisarmos de forma setorial e conjuntural como tem sido a realidade contemporânea, para além do acometimento pelo vírus em si. Assim, este estudo tem como objetivo refletir criticamente o cenário da situação de saúde de pessoas LGBTI+ frente a Covid-19 em contexto de pandemia no Brasil, sob o enquadramento teórico de Michel Foucault ${ }^{(4-6)}$ e Judith Butler ${ }^{(7-8)}$.

\section{Método}

Estudo teórico-reflexivo, com abordagem qualitativa, estruturado a partir do arcabouço 
teórico e analítico advindos dos campos dos estudos queer no qual será acionado os conceitos de dispositivo da sexualidade e biopolítica elaborados por Michel Foucault ${ }^{(5-6)}$ e os de gênero, heteronormatividade e a noção de vida precária formulados por Judith Butler ${ }^{(7-8)}$. Destarte, o diálogo com esse referencial teórico facultou a compreensão de achados empíricos sobre a pandemia obtidos a partir da estruturação do estudo, por meio da realização da decomposição não estruturada e aleatória dos achados publicados, no período de 10 a 20 de abril de 2020, em documentos oficiais produzidos por entidades civis organizadas e movimentos sociais.

O material foi coletado em fontes disponíveis, ambiência virtual e em artigos científicos indexados nas bases de dados científicos como a Lilacs, Medline, Pubmeb e Web of Science, bem como em bibliotecas como a Scielo, selecionados a partir do emprego de busca livre em utilização aos descritores controlados na plataforma DeCs- Descritores em Ciências da Saúde e MeSh - Medical Subject Headings da NCBI, relacionados à Covid-19 e a interseção com palavras chaves e sinônimos relacionados à gênero e à saúde de pessoas LGBTI+, especialmente no Brasil.

Como a proposta desse estudo foi tecer in(re)flexões acerca da temática em estreita relação com a abordagem qualitativa, tendo em vista a interpretação e a análise dos elementos teóricos obtidos por meio do levantamento bibliográfico, optou-se por não apresentar um quadro-síntese sobre o estado da arte.

No que se refere aos aspectos éticos, excluiu-se a necessidade de submissão a Plataforma Brasil para apreciação ética em virtude de não se tratar de uma pesquisa direta/aplicada envolvendo seres humanos. No entanto, cumpriu-se em todas as etapas de elaboração do estudo os preceitos éticos, em especial o da beneficência, não-maleficência, confiabilidade e a veracidade dos dados produzidos. Como forma de garantir o rigor e a qualidade na pesquisa científica, adotou-se as diretrizes propostas no Guideline SQUIRE 2.0.

\section{Resultados e Discussão}

Dentre as dificuldades cotidianas encontradas nas histórias de vidas de pessoas LGBTI+, tem-se que os espaços de sociabilidade doméstica muitas vezes funcionam como lócus referencial de potencialização de vulnerabilidade(s), sobreposição de violência(s) e exclusão. Por quê? Porque é a partir da "não aceitação", reconhecimento e respeito à orientação sexual e identidade de gênero por parte da família, que LGBTI+ passam a enfrentar uma miríade de violência física, insultos, violação de direitos e negação existencial. Por conseguinte, os momentos da escola, na faculdade, no trabalho, e em atividades diversas assim como em possíveis cenários de não pandemia, são cruciais para pensarmos as dinâmicas de subordinação à trama violenta que é o compartilhar de espaços e momentos com os elementos que ainda abusam, fazem sofrer, adoecem, quando não exterminam, essas vidas que, como todas as vidas, importam. Quando essas válvulas de escape (momentos extra domésticos) não podem ser acionadas, o que resta?

Em se tratando da(s) experiência(s) pessoas transgêneras podem somar às experiências de vulnerabilidade doméstica, outras relacionadas ao trabalho, em grande parte informal, tendo em vista que, como tem ocorrido no Brasil, há certa dificuldade, por parte do Governo Federal no que se refere ao reconhecimento e legitimidade das necessidades de determinadas categorias, no recebimento do auxílio emergencial. O que a ausência deste auxílio determina? Mais vulnerabilidade social e disposição para situações de riscos, uma vez que não trabalhar está relacionado com a falta de manutenção de questões básica para sobrevivência ${ }^{(9)}$. Tal problemática tem exposto mulheres transgêneras e travestis ao trabalho sexual mesmo durante o período em que a maior parte das cidades e municípios tem a quarentena e/ou o lockdown mantidos ${ }^{(10)}$. Desse modo, entre a difícil escolha existente em se manter protegida contra o vírus e ter a sua subsistência garantida, essas mulheres não tem grandes saídas frente as desigualdades e a precarização do trabalho sexual face a pandemia ${ }^{(11)}$. 
Para além disso, a devastação que é carregada de negligências, invisibilidades e iniquidades gera repercussão ainda maior, ao passo que as cenas de entretenimento drag, transformista e LBGTI+ são fortemente afetadas com a interrupção abrupta dos eventos que movimenta grande capital financeiro no país e que é responsável por garantir o trabalho, embora em grande número informal ${ }^{(12)}$.

Outra faceta que a experiência de pessoas transgêneras pode encontrar, está relacionada com a legalidade jurídica, como ocorreu no Panamá e no Peru, desde que o governo local decidiu por determinar dias alternados de saída pública para homens e mulheres, não reconhecendo as pessoas transgêneras em nenhuma dessas identidades de gênero ${ }^{(13)}$. Posicionamentos como esses tornam as vidas das pessoas transgêneras impossíveis de serem vividas - em termos butlerianos. Frente a essas (im)posições, quais são as reais possibilidades de manutenção da vida em que pese as condições precárias? Assim, quando a pessoa transgênera deixa de ser reconhecida como pessoa, nos remete ao que Foucault denominou como regime de verdade, ao problematizar como estas se constituem em uma época ${ }^{(6)}$. Nesses casos, sob essas condições, quem realmente pode? A esse respeito, Butler reitera que os regimes de verdade facultam o reconhecimento de si e do mundo, mas que não se trata de quadros imutáveis, pois o horizonte normativo é alvo de uma abertura crítica a partir do modo como sou vista/o e/ou percebida/o e o modo como vejo/percebo a/o outra/o ${ }^{(8)}$.

No Brasil, apesar dos avanços e conquistas do movimento social organizado de pessoas transgêneras, no que se refere a políticas públicas e direitos humanos, como o processo transexualizador e o uso do nome social - a retificação do nome civil sem a necessidade de ajuizar uma ação - observa-se que o cuidado e o tratamento em saúde ainda permanecem restrito as conformações corporais, como se essa fosse a principal e única demanda de todas as pessoas.

Ainda sobre garantias públicas, e mais especificamente no âmbito da atenção à saúde, é relevante destacar as múltiplas dimensões a serem levadas em consideração quanto aos impactos gerados à população LGBTI+ durante e pós-pandemia. Diante do cenário de assimetrias, violências, estigmatização, preconceito e discriminação à população LGBTI+, teme-se que a oferta de tecnologias cuidativas disponibilizadas no Sistema Único de Saúde (SUS) possam ser atingidas, gerando efeitos deletérios para a qualidade de vida e saúde de pessoas LGBTI+ que, por exemplo, convivem com o HIV e outras Infecções Sexualmente Transmissíveis (IST) e de tantas outras que são beneficiadas com ações e programas, a exemplo da Profilaxia Pré-Exposição (PreP) e Profilaxia Pós-Exposição (PEP) para HIV, a oferta de preservativos, gel lubrificante, a realização de testes rápidos, inclusive para a população adolescente $^{(14)}$. Igualmente, daquelas/es que necessitam acessar a hormonioterapia, como as travestis, mulheres e os homens trans e da cobertura de ações de prevenção de danos e riscos à saúde e ao enfrentamento à violência ${ }^{(15)}$.

No âmbito da saúde à mulher, temos as mulheres bissexuais e lésbicas, em grande parte, ainda invisibilizadas no que se refere as ações de promoção à saúde da população LGBTI+. Há que se preocupar com a proteção e a segurança contra as violências desse grupo, assim como a garantia dos direitos sexuais e reprodutivos, uma vez que se prevê que cerca de 18 milhões de mulheres possam ficar sem acesso aos métodos contraceptivos sendo a interrupção legal da gravidez (em caso de estupro ou em situações em que haja risco de vida para a gestante) vista como a necessidade de real atenção ginecológica, incluindo cirurgias e outras tecnologias de cuidado essenciais que extrapolam essa dimensão ${ }^{(16)}$. No tocante a violência, chama-se à atenção para um aspecto importante e pouco discutido com vistas a desmitificação, como se as relações lésbicas estivessem livres de violência, pelo fato de serem mulheres. Entretanto, na medida em que não se discute coletivamente sobre o assunto poderá retroalimentar uma cultura de silêncio e isolamento quando uma mulher é vítima da violência perpetrada por outra mulher ${ }^{(17)}$.

Tal impacto também pode prejudicar a garantia da atenção psicossocial nos diferentes 
aparelhos do sistema, em razão do elevado adoecimento mental, que pode alargar-se, mediante ao estresse pós-traumático ocasionado pela pandemia e da agudização de outras disparidades e limitações no acesso aos serviços de saúde.

A realidade das populações LGBTI+ em sua condição de vida precária é reflexo de um longo processo histórico de vigência da biopolítica heteronormativa, tal como formulado pelo filósofo Michel Foucault. Em seus estudos, Foucault afirma que a sexualidade é um dispositivo histórico de saber-poder em nome da qual se legitimam e viabilizam práticas autoritárias de segregação, monitoramento, gestão dos corpos e do desejo, utilizando a figura do casal heterossexual como padrão de normalidade, o qual também foi o elemento basilar para a produção de uma biopolítica dirigida ao "governo sobre a vida" (4-5)

Ampliando essa noção, a filósofa Judith Butler menciona a formação de um regime heteronormativo que dá significação aos corpos, gêneros e desejos, a partir do processo de normalização apontado por Michel Foucault, sendo que os seres assimilados pela heteronormatividade como anormais são situados nas zonas inóspitas e inabitáveis da vida social, operando assim as desigualdades no acesso aos bens materiais e simbólicos, produtora das vulnerabilidades que tornam a vida das populações não heterossexuais não vivíveis, podendo, então, serem deixadas à morte $e^{(4-5,7)}$.

Essas reflexões nos ajudam a pensar que as negligências e as violências que produzem a vulnerabilidade das populações LGBTI+ são efeitos desse saber-poder-verdade que regula os corpos a partir da biopolítica heteronormativa em nossa sociedade. No contexto de enfrentamento de uma pandemia, a condição de vida precária operará formas de exposição e letalidade dessa população que terá sua vulnerabilidade potencializada na dimensão de uma biopolítica voltada ao controle da disseminação do contágio ao Covid-19.

Um ensaio recente publicado no livro Sopa de Wuhan, Judith Butler observa que no momento em que vivemos o vírus por si só não discrimina, mas as desigualdades são operadas em nossa sociedade no processo histórico que entrelaçou o nacionalismo com a pobreza, o racismo, a xenofobia e o capitalismo, de modo que esta dinâmica promove uma acentuação na distinção espúria entre vidas passíveis e não passíveis de luto, onde a produção da precariedade elege as formas de vidas que estão protegidas contra a morte e aquelas cujas vidas são consideradas não valerem o bastante para serem salvaguardadas contra a doença e a morte ${ }^{(18)}$.

Com base nesse panorama em que os fatos apresentados revelam a sobreposição de vulnerabilidades, questiona-se: quantas são as pessoas LGBTI+ afetadas pela Covid-19 no Brasil? Marcadores relacionados à identidade de gênero têm sido considerados nas notificações de agravos à saúde? Ações programáticas e estratégias têm sido regadas a fim de garantir a minimização dos impactos? A implementação das ações da Política Nacional de Atenção Integral à Saúde da População LGBT tem sido operacionalizada? ${ }^{(19)}$.

Sob esse prisma, também preocupada com à saúde da população LGBTI+ diante desse cenário, a Associação Brasileira de Saúde Coletiva (Abrasco), emitiu uma nota tecendo considerações que conclamam para a quebra do silêncio e a invisibilidade destinada por diversos órgãos e instituições, como o Estado e a sociedade civil, a pautarem o assunto como uma agenda essencial na saúde pública no Brasil e no restante do mundo $^{(20)}$. Há que se inferir sobre a necessidade de uma mudança real nos paradigmas existentes no âmbito teórico e organizacional das relações cuidativas no contexto da produção do cuidado à saúde de pessoas LGBTI+.

Um outro campo a ser discutido é o lugar dos aplicativos digitais dedicados à promoção de relacionamentos sociais, utilizados em grande número por homens gays, bissexuais, pessoas transgêneras e homens que fazem sexo com homens (HSH). Convém ressaltar que as plataformas de encontros virtuais sob a óptica de usuários, tem feito parte de maneira mais veemente da vida cotidiana das pessoas que fazem uso desses dispositivos. Nesse aspecto, se o momento pede distanciamento e isolamento social, encontros físicos ocasionados por meios 
dessas plataformas podem pôr em risco a saúde dos seus usuários, constituindo em mais um aspecto a ser observado. A este respeito, as ações com vistas a preservação da vida desses usuários devem fazer parte da agenda dos gestores dessas tecnologias através da propagação de chamadas de educação e comunicação em saúde, como forma de contribuir com a diminuição do contágio, caso o contato físico seja mantido ${ }^{(21-23)}$.

Considerando tais contextos problematizadores, enfatiza-se a emergência em considerar como uma questão prioritária as dimensões relacionais de gênero e, por consequência, as masculinidades e feminilidades, assim como a não binaridade de gênero na promoção das ações relacionadas ao enfrentamento da Covid-19 ${ }^{(24-25)}$. Tal panorama deve-se tornar-se efetivo não só no Brasil, mas em todo o planeta, como reconheceu recentemente a Organização Mundial da Saúde ao publicar uma nota na $73^{\circ}$ Conferência Mundial de Saúde sobre a relevância do marcador de gênero no enfrentamento à pandemia ${ }^{(26)}$.

Por fim, outro fator potencializador de prejuízos as vidas das pessoas LGBTI+ no Brasil, durante a pandemia, são os ataques cotidianos gerados pela representação presidencial aos direitos desse segmento populacional, em que pese o constante potencial gerador de estresse ao bem-viver de pessoas que (con)vivem com a violação de direitos humanos básicos, bem como o posicionamento negacionista, obscurantista, reacionário, LGBTfóbico e deslegitimador da(s) existência(s) de pessoas LGBTI+ tal qual o Brasil têm sido reconhecido internacionalmente ${ }^{(27)}$.

\section{Conclusão}

O estudo refletiu teoricamente questões iniciais sobre a dimensão da saúde de pessoas LGBTI+ a partir da ocorrência da pandemia em curso do novo Coronavírus (SARS-CoV-2) causador da Covid-19 no Brasil, e elucida de forma denunciadora os aspectos geradores de impactos e repercussões negativas que intensificam e sobrepõe desigualdades, vulnerabilidades que se revertem em iniquidades em saúde. Destarte, chama-se a atenção de que o enfrentamento e a superação dos impactos provocados pelo contexto pandêmico devem validar a sobrevida de todas as pessoas e não apenas de algumas.

Repensar as práticas de segregação, discriminação, violências e preconceitos as quais a biopolítica expõe determinados grupos humanos, como ocorre com as pessoas LGBTI+, fará com que haja emergente necessidade de assegurar a proteção de todas as vidas vivíveis. Especificamente no campo da atenção à saúde, é relevante pensar o enfrentamento da pandemia associado a intervenção de medidas de correção das desigualdades na oferta de serviço, que tem potencial expressivo de precarização das vidas.

Embora o estudo limite-se em aportar um cenário inicial, sem abrangência metodológica empírica, tal como a carência de estudos indexados em bases brasileiras e internacionais sobre o tema, fatos elucidados nesta reflexão desvelam a magnitude de uma problemática que já é real e ocasionadora de prejuízos à saúde e a condição humana. Com base nisso, recomenda-se o aprofundamento de investigações sobre a problemática refletida, como forma de ampliar o conhecimento sobre o fenômeno e consequentemente suscitar intervenções eficazes a serem implementadas no cotidiano das práticas em todo o território nacional e demais contextos planetários.

\section{Colaborações:}

1 - concepção, projeto, análise e interpretação dos dados: Anderson Reis Sousa e Caio Felipe Campos Cerqueira;

2 - redação do artigo e revisão crítica relevante do conteúdo intelectual: Carle Porcino e Kleber José Fonseca Simões;

3 - aprovação final da versão a ser publicada: Anderson Reis Sousa e Kleber José Fonseca Simões.

\section{Referências}

1. Barreto ML, Barros AJD, Carvalho MS, Codeço CT, Hallal PRC, Medronho RA et al. What is urgent 
and necessary to inform policies to deal with the COVID-19 pandemic in Brazil? Rev bras. epidemiol. 2020; 23: e200032. DOI: https://doi. org/10.1590/1980-549720200032

2. Antunes L. Coronavírus: o que pessoas LGBT+ precisam saber para preservar sua saúde [Internet]. O globo, 2020 [cited 2020 May 15]. Available from: https://oglobo.globo.com/celina/coronavirusque-pessoas-lgbt-precisam-saber-para-preservarsua-saude-1-24354788

3. OutRight Action International. Vulnerability amplified: The Impact of the COVID-19 Pandemic on LGBTIQ People. New York: OutRight Action International, 2020 [cited 2020 May 15]. Available from: https://outrightinternational.org/sites/default/ files/COVIDsReportDesign_FINAL_LR_0.pdf

4. Foucault M. Em defesa da sociedade: curso do Collège de France (1975-1976). São Paulo: Martins Fontes; 1999.

5. Foucault M. História da sexualidade I: o cuidado de si. 10. ed. Rio de Janeiro: Edições Graal; 1988.

6. Foucault M. Malfazer, dizer verdadeiro: função da confissão em juízo - curso em Louvain, 1981. 1a ed. São Paulo: WMF Martins Fontes; 2018.

7. Butler J. Corpos que pesam: sobre os limites discursivos do "sexo". In: Louro GL, organizadora. O corpo educado: pedagogias da sexualidade. $4 \mathrm{a}$ ed. Belo Horizonte: Autêntica Editora; 2018.

8. Butler J. Relatar a si mesmo: crítica da violência ética. Belo Horizonte: Autêntica; 2017.

9. Silva AS, Luna MS. Travestis e transgêneros e sua inserção no mercado formal de trabalho. Cad Gên Tecnol. 2019; 12(39):303-318. DOI: 10.3895/cgt. v12n39.9506

10. Huffpost Brasil. "Pessoas trans ficam em 'limbo jurídico' por medidas de isolamento na América Latina" [Internet]. 2020 [cited 2020 May 15]. Available from: https://www.huffpostbrasil. com/entry/pessoas-trans-coronavirus_ br_5e876739c5b609ebfff0835f?ncid=other_ huffpostre_pqylmel2bk8\&utm_campaign=related_ articles

11. Fernandes Y. Prostituição e pandemia: 'Terei que aceitar 20 ou 30 reais, preciso comer [Internet]. Portal colabora, 2020 [cited 2020 May 15]. Available from: https://projetocolabora.com.br/ ods8/prostituicao-e-pandemia-terei-que-aceitar20-ou-30-reais-preciso-comer/

12. Bonis, B. Coronavírus: boates de Berlim tentam sobreviver com baladas 'virtuais' [Internet].
BBC News, 2020 [cited 2020 May 15]. Available from:https://www.bbc.com/portuguese/internacional52178060

13. Benevides B. Panamá e Peru violam direitos de trans durante a quarentena ao coronavírus [Internet]. Revista hibrida, 2020 [cited 2020 May 15]. Available from: https://revistahibrida.com.br/ 2020/04/10/panama-e-peru-violam-direitos-transdurante-quarentena-do-coronavirus/

14. Dourado I, Magno L, Soares F, Massa P, Amy Nunn A, Shona Dalal S et al.. Adapting to the COVID-19 Pandemic: Continuing HIV Prevention Services for Adolescents Through Telemonitoring, Brazil. AIDS Behav., 2020; 24:1-6. DOI: 10.1007/ s10461-020-02927-w

15. Sousa D, Iriart J. "Living with dignity": health needs and demands of trans men in Salvador, Bahia State, Brazil. Cad Saúde Pública. 2018; 34(10): e00036318. DOI: http://dx.doi. org/10.1590/0102-311X00036318

16. Bruno MM. Como vão os direitos reprodutivos na América Latina durante a pandemia do coronavírus [Internet]. Genero número, 2020 [cited 2020 May 15]. Available from: http://www. generonumero.media/como-estao-os-direitosreprodutivos-na-america-latina-em-tempos-decoronavirus/

17. Cabral REG. Escrever-se travesti, reescrever-se sapatão: um recorrido sobre corpos e afetividades insubmissas. In: Soares MR, Brandão S, Faria T (org). Lesbianidades plurais: abordagens e espistemologias sapatonas. Salvador: Devires; 2019.

18. Agamben G, Zizek S, Nancy JL, Berardi FB, Petit SL, Butler J et al. Sopa de Wuhan. $1^{\underline{a}}$ Ed. ASPO (Aislamiento Social Preventivo y Obligatorio); 2020.188p

19. Brasil. Ministério da Saúde. Política Nacional de Saúde Integral de Lésbicas, Gays, Bissexuais, Travestis e Transexuais - LGBT. Brasília: Ministério da Saúde, 2008

20. Associação Brasileira de Saúde Coletiva. Considerações da Abrasco sobre a saúde da população LGBTI+ no contexto da epidemia de Covid-19 [Internet]. 2020 [cited 2020 May 15]. Available from: https://www.abrasco.org.br/ site/noticias/posicionamentos-oficiais-abrasco/ consideracoes-da-abrasco-sobre-a-saude-dapopulacao-lgbti-no-contexto-da-epidemia-decovid-19/47257/ 
21. Belém JM, Alves MJH, Pereira EV, Moreira FTLS, Quirino GS, Albuquerque GA. Atenção à saúde de Lésbicas, Gays, Bissexuais, Travestis e Transexuais na Estratégia Saúde da Família. Rev baiana enferm. 2018; 32: e26475. DOI: 10.18471/rbe.v32.26475

22. Argentina. Nuevo coronavirus covid-19. información, recomendaciones del Ministerio de la Salud de la Nación y medidas de prevención [Internet]. 2020 [cited 2020 May 15]. Available from: https://www.argentina.gob.ar/salud/ coronavirus-COVID-19

23. NYC Health. Sex and Coronavirus Disease 2019 (COVID-19). NYC. Health. 2020 [cited 2020 May 15]. Available from: https://www1.nyc.gov/ assets/doh/downloads/pdf/imm/covid-sexguidance.pdf

24. Lancet. The gendered dimensions of COVID-19. Lancet. 2020; 395(10231):1168. DOI: 10.1016/ S0140-6736(20)30823-0
25. Ewig C. Gender, Masculinity, and COVID-19 [Internet]. The Gender Policy Report, 2020 [cited 2020 May 15]. Available from: https://genderpolicyreport.umn.edu/gendermasculinity-and-covid-19/

26. Dias S. Aplicativos de pegação gay emitem comunicado sobre coronavírus [Internet]. Observatório G, 2020 [cited 2020 May 15]. Available from: https://observatoriog.bol.uol.com. br/noticias/2020/03/aplicativos-de-pegacao-gayemitem-comunicado-sobre-coronavirus

27. Lancet. COVID-1.9. in Brazil: "So what?". The lancet. 2020; 395(10235):1461. DOI: https:// doi.org/10.1016/S0140-6736(20)31095-3

Recebido: 24 de maio de 2020

Aprovado: 20 de junho de 2020

Publicado: 23 de novembro de 2020

A Revista Baiana de Enfermagem utiliza a Licença Creative Commons - Atribuição-NãoComercial 4.0 Internacional. https://creativecommons.org/licenses/by-nc/4.0/

Este artigo é de acesso aberto distribuído sob os termos da Licença Creative Commons (CC BY-NC). Esta licença permite que outros remixem, adaptem e criem a partir do seu trabalho para fins não comerciais. Embora os novos trabalhos tenham de lhe atribuir o devido crédito e não possam ser usados para fins comerciais, os usuários não têm de licenciar esses trabalhos derivados sob os mesmos termos. 